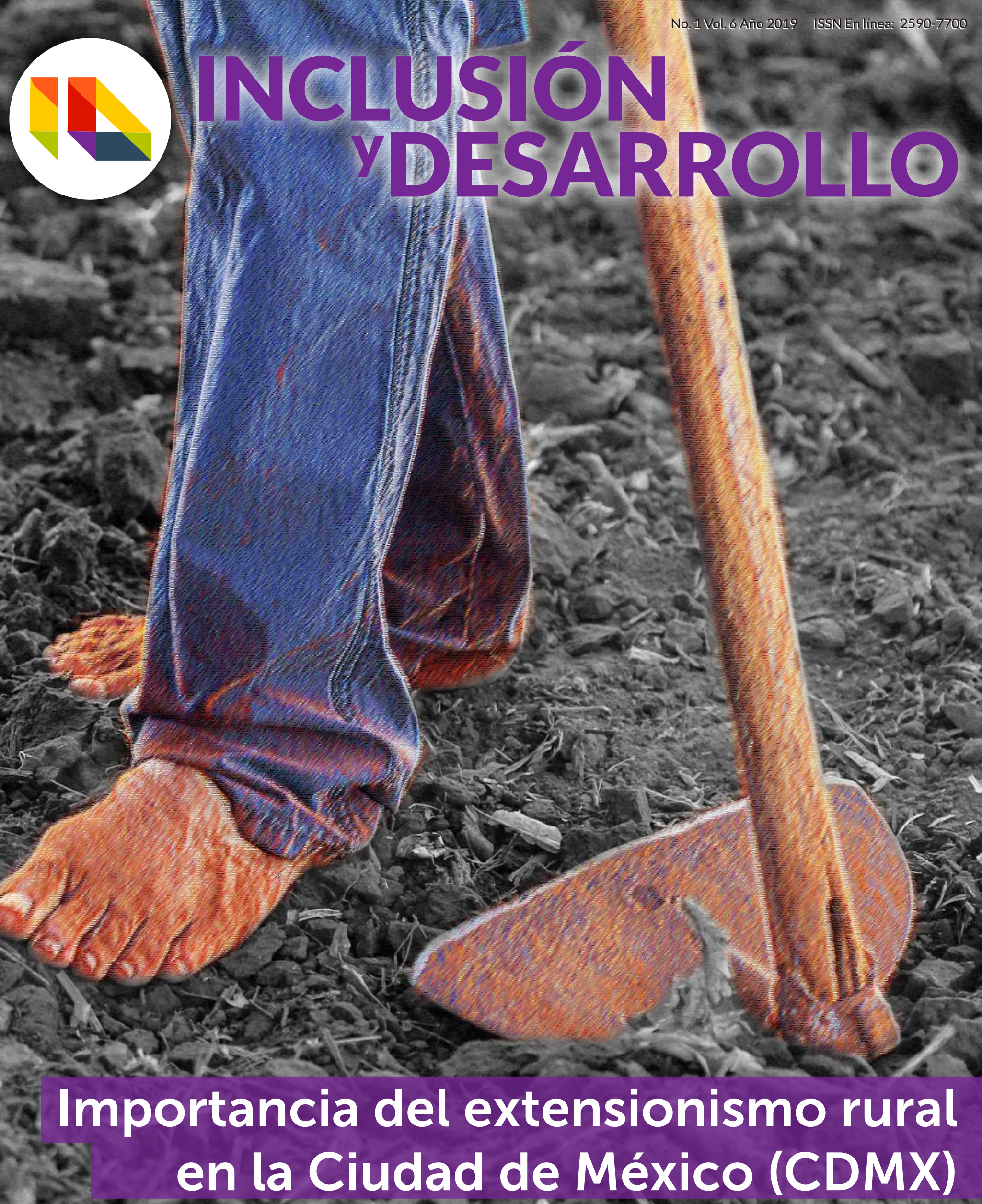

Georgel Moctezuma López, Eric Uriel Ramírez Sánchez, Lourdes Velázquez Fragoso y Alejandra Vélez Izquierdo 


\title{
IMPORTANCIA DEL EXTENSIONISMO RURAL en la CIUDAd de MÉxico (CDMX)
}

\section{RURAL EXTENSIONISM IMPORTANCE at MeXico City (CDMX)}

\author{
Georgel Moctezuma López \\ moctezuma.georgel@inifap.gob.mx \\ Centro Nacional de Investigación Disciplinaria \\ en Conservación y Mejoramiento de Ecosistemas Forestales \\ México D.F-México
}

Eric Uriel Ramírez Sánchez extensionismo.cdmx@inifap.gob.mx Universidad Nacional Autónoma de México Ciudad de México - México

Lourdes Velázquez Fragoso extensionismo.cdmx@inifap.gob.mx Universidad Nacional Autónoma de México

Ciudad de México - México

Alejandra Vélez Izquierdo velez.alejandra@inifap.gob.mx Centro Nacional de Investigación Disciplinaria en Fisiología y Mejoramiento Animal. INIFAP. Ajuchitlán, Qro. - México

\section{Resumen}

Objetivo. Analizar diversos componentes que participaron en el proyecto que se conoce como Apoyo al Extensionismo Rural, Desarrollo de Capacidades y Asociatividad Productiva de la SAGARPA y que se compartió en el año 2016, 2017 y 2018 junto con el Gobierno de la CDMX por medio de la SEDEREC.

Metodología. Por medio de una interacción participativa se sumaron al componente de extensionismo diversas instituciones entre las cuales se puede mencionar al INCA Rural, la UACh, la CONAPESCA y el INIFAP; así mismo en su fase intermedia se sumaron instituciones educativas superiores como el Instituto Tecnológico de Milpa Alta, la UAM en sus campus Xochimilco e Iztapalapa, la UNAM por conducto de la FES Aragón y la Facultad de Medicina Veterinaria. La idea central fue la de evaluar el componente de extensionismo agrícola en la CDMX por medio de una encuesta que se aplicó a los productores de las cinco cadenas agroproductivas que se seleccionaron: amaranto, maíz, nopal, hortalizas y ornamentales, todas ellas representativas de la actividad agrícola de la CDMX.

Originalidad. Las acciones de asesoría y acompañamiento técnico se realizaron fundamentalmente en las cuatro Delegaciones del sureste de la Ciudad de México: Milpa Alta, Tláhuac, Tlalpan y Xochimilco.

Conclusión. Se detectaron las principales innovaciones tecnológicas que se implementaron bajo la conducción de los extensionistas, así como los distintos tipos de beneficio que se generaron con las innovaciones (Moctezuma et al., 2018).

Palabras clave: extensionismo, cadenas, innovaciones, evaluación y asesoría técnica. 


\section{Abstract}

Objective. To analyze various components that participated in the project known as rural extensionism Support, development of Capacities and Productive Associativity of SAGARPA and that was shared in 2016, 2017 and 2018 with the Government of CDMX through SEDEREC.

Methodology. Through a participatoryinteraction, various institutions were added to the extensionism component, withing which we can mention INCA Rural, UACh, CONAPESCA and INIFAP; Likewise, in its intermediate phase, higher educational institutions such as the Technological Institute of Milpa Alta, the UAM in its Xochimilco and Iztapalapa campuses, the UNAM through the FES Aragón and the Faculty of Veterinary Medicine joined. The central idea was to evaluate the component of agricultural extensionism in the CDMX by means of a survey that was applied to the producers of the five agricultural production chains that were selected: amaranth, corn, cactus, vegetables and ornamentals, all representative of the agricultural activity of the CDMX.

Results. The advisory and technical support actions were carried out mainly in the four Delegations of the southeast of Mexico City: Milpa Alta, Tláhuac, Tlalpan and Xochimilco.

Conclusion. The main technological innovations that were implemented under the management of the extension agents were detected, as well as the different types of benefits that were generated with the innovations (Moctezuma et al., 2018).

Keywords: extensionism, chains, innovations, evaluation and technical advisory. 


\section{INTRODUCCIÓN}

a palabra extensionismo tiene su origen en la - palabra latina extensio, onis, que significa la acción y efecto de extender o extenderse (DRAE, 2016). Por otro lado, la Secretaría de Agricultura, Ganadería, Desarrollo Rural, Pesca y Alimentación (SAGARPA), dentro de su portal SERMEXICANO (2016) (Mcmahon et al., 2011) (Sanderson 1990) (Rendón et al., 2015) lo define como "el servicio prestado por personal de las instituciones de educación y de investigación que facilita el acceso al conocimiento, la información y las tecnologías, a productores, grupos y organizaciones económicas rurales y a otros actores del sector agropecuario, pesquero y acuícola". La importancia del extensionismo agrícola en la Ciudad de México (CDMX) radica fundamentalmente en el enorme mercado de casi nueve millones de habitantes (CONAPO 2015) que demandan una gran cantidad de productos alimenticios del sector agrícola. Durante el periodo 2000 - 2015, la tasa media de crecimiento anual para la población de la CDMX fue de 0.90\% que es de las más bajas en la república mexicana y en contraste con lo anterior. La superficie sembrada en la CDMX para el mismo periodo presenta una disminución en su tasa media de crecimiento anual de $-2.75 \%$, lo cual hizo que en términos absolutos pasara de aproximadamente 27,000 hectáreas a 17,500 hectáreas (INEGI 2015) (SIAP 2013, 2014 y 2015), que en buena medida se debe a la presión de la mancha urbana. Ésta situación ambivalente, de que, por un lado, en términos absolutos se tenga un aumento población de casi tres millones de personas en un plazo de 15 años y de que por el otro se reduzca la superficie agrícola que se destina al suministro de alimentos, hace que la CDMX no sea autosuficiente, y con mucho, en autoabastecerse, hace que sea un importador neto de alimentos provenientes de todos los estados de la república mexicana (Moctezuma et al., 2008) (Moctezuma et al., 2016)

Resulta importante hacer énfasis en que la CDMX (antes Distrito Federal) es el corazón de los Estados Unidos Mexicanos, ya que en ella se asientan los poderes ejecutivo, legislativo y judicial del país y su origen se remonta a la gran Tenochtitlán que se fundó en 1325 (Wikipedia 2016), (Reyes 2013) (Cadena et al., 2009) (Cadena et al., 2013). Tenochtitlán fue una gran zona productora agrícola e hizo uno de los aportes más grandes al desarrollo rural por medio de la tecnología que se conoció como la de producción en chinampas, las cuales eran espacios de cultivos dentro del lago y fueron autosuficientes para abastecer a la población azteca (Cuevas 2012 y 2013).

La CDMX es el estado más pequeño del país, ya que tiene una superficie de $1,485 \mathrm{Km} 2$, lo cual representa apenas el $0.08 \%$ de la superficie total de México, con una división política compuesta por 16 alcaldías (antes delegaciones) como se muestra en el Cuadro 1.

Cuadro 1. Alcaldías (antes Delegaciones) de la CDMX y superficie en Km2

\begin{tabular}{|c|c|c|c|}
\hline Nombre & Superficie & Nombre & Superficie \\
\hline Álvaro Obregón & 96.17 & Iztapalapa & 717.00 \\
\hline Azcapotzalco & 33.66 & Magdalena Contreras & 44.58 \\
\hline Benito Juárez & 26.63 & Miguel Hidalgo & 228.41 \\
\hline Coyoacán & 54.40 & Milpa Alta & 85.34 \\
\hline Cuajimalpa & 74.58 & Tláhuac & 312.00 \\
\hline Cuauhtémoc & 32.4 & Tlalpan & 33.40 \\
\hline Gustavo A. Madero & 94.07 & Venustiano Carranza & 122.00 \\
\hline Iztacalco & 22.30 & Xochimilco & \\
\hline
\end{tabular}

Fuente: INEGI. 2014 
Las alcaldías más grandes territorialmente son en primer lugar Tlalpan, sigue Milpa Alta y en tercer lugar Xochimilco, mismas que porcentualmente ocupan el $21 \%, 15.4 \%$ y $8,2 \%$ respectivamente, entre ellas suman el $44.6 \%$ de la superficie de la CDMX, en éstas alcaldías es en donde se concentra la actividad agropecuaria y si se le suma la delegación Tláhuac, que también tiene una fuerte actividad primaria, la superficie alcanza prácticamente la mitad (50.3\%) de la superficie de la CDMX, alcaldías en donde se focaliza el programa de extensionismo (Villareal 2015) (Zamora 2016).

Dentro de los procesos de PS\&E (planeación, seguimiento y evaluación) es menester cuantificar el grado de satisfacción de los beneficiarios del componente extensionismo (agricultores, ganaderos y acuicultores), por lo que, se planteó el hecho de sí los productores deseaban que el servicio de asesoría facilitado por extensionistas de la SAGARPA y la SEDEREC, se debía o no, continuar y fue así que se fijó el objetivo del presente trabajo como el de evaluar el programa de extensionismo agrícola dirigido a pequeños productores dentro de los niveles I y II de la CDMX, ejecutado por extensionistas y con el acompañamiento de universidades (Universidad Autónoma Chapingo) y centros de investigación (INIFAP), para a su vez presentarlo ante las autoridades federales de la SAGARPA y estatales del Gobierno de la CDMX.

\section{Metodología}

El proceso metodológico que se utilizó para la evaluación del componente extensionismo fue el de tomar en cuenta las acciones propuestas dentro del proyecto de Apoyo al Extensionismo Rural de la SAGARPA, (DOF 2103) (DOF 2015) en el cual el INIFAP tuvo como actividad principal la de acompañamiento tecnológico, la cual consistió de las siguientes fases:

Selección de los extensionistas, para la incorporación del personal técnico, la SAGARPA en coordinación con la Secretará de Desarrollo Rural y Equidad de las Comunidades (SEDEREC) del Gobierno de la CDMX lanzaron una convocatoria abierta al público para recibir a los candidatos y por medio de un proceso de puntuación (scoring) se preseleccionaron candidatos para posteriormente, sumar el resultado de sus entrevistas y elegir a los mejores técnicos agrícolas, así como a la coordinadora de extensionistas (Vázquez y Landa 2017).

Selección de cadenas de valor, con el propósito de cuantificar y dimensionar la importancia de la actividad agrícola dentro de la CDMX, hizo la consulta del Plan Estratégico (SAGARPA 2007) que elaboró la SAGARPA y SEDEREC con la conducción del INCA Rural, del cual se extrajeron las cadenas agroproductivas más relevantes de la CDMX que se consideraron dentro del proyecto de Apoyo al Extensionismo Rural de la SAGARPA.

Elaboración de las agendas de innovación y programas de trabajo de los extensionistas a nivel individual y por cadena, mediante un guion predeterminado y con la participación de la Universidad Autónoma Chapingo (Manrubio et al., 2007) (Villareal 2015) se realizaron una serie de talleres participativos con los extensionistas para definir las acciones que se consideraron en las agendas; éstas sirvieron de marco normativo a los técnicos para que cada uno de ellos integrará su programa de trabajo, mismo que debía estar alineado a la agenda de innovación de la cadena.

Socialización de las agendas de innovación, para esta actividad se realizaron talleres por cada una de las cadenas agroproductivas que se seleccionaron, los actores principales de éstos talleres fueron los productores líderes, productores cooperantes y productores acompañantes a los cuáles se les explicó en qué consistió y como se elaboró la agenda para que en una segunda fase hicieran su retroalimentación y aportes, los cuales se incorporaron a las agendas de innovación para su validación y sobre todo, su apropiación (Aguilar et al., 2010) (Saldaña et al., 2006).

Implementación de las innovaciones, se realizaron in situ bajo la supervisión directa de cada uno de los extensionistas responsables y con visitas periódicas a sus predios con la finalidad de observar cual fue la evolución de las innovaciones planteadas (Turrent 20017).

Recorridos de campo de supervisión, se realizaron visitas de supervisión por parte de las tres instituciones encargadas de ésta actividad: Centro de Extensión e Innovación Rural de la Universidad Autónoma Chapingo, el INCA Rural y el INIFAP, así como también del acompañamiento de funcionarios de la Delegación Estatal CDMX de la SAGARPA y de la SEDEREC del Gobierno de la CDMX. 
Evaluación del componente extensionismo, para la valoración del programa se diseñó un cuestionario para que fueran contestados por parte de los productores dedicados a la actividad agrícola, con dos grupos de preguntas; uno relativo a preguntas cualitativas y el otro de tipo cuantitativo, en total de preguntas fue de 16 , de las cuales 10 fueron cualitativas y cinco de tipo cuantitativo. El tamaño de la muestra se determinó de acuerdo a los estratos dentro del universo de productores; se aplicó muestreo aleatorio estratificado con tamaño de muestra de fijación proporcional (Gregoire and Valentine 2008) bajo la fórmula:

$$
N=\sum_{h=1}^{L} N_{h}
$$

En donde:

$\mathrm{N}$ = Población

$\mathrm{L}=$ Estratos

$\mathrm{Nh}=$ unidades de cada uno de los L estratos

Con lo anterior tenemos:

$n h=n\left(\frac{N_{h}}{N}\right)=n W_{h}$

En la cual:

$\mathrm{nh}=$ número de muestra del estrato

$\mathrm{n}=$ número de muestras

$\mathrm{N}=$ población

$\mathrm{n}=\mathrm{nWh}=$ número de muestras ponderadas por estrato

De lo anterior, la aplicación de los datos se muestra en el Cuadro No. 2

Cuadro 2. Distribución de datos de los productores participantes por cadena de valor en el componente extensionismo rural, desarrollo de capacidades y asociatividad productiva de la CDMX.

\begin{tabular}{|c|c|c|c|c|c|c|c|c|c|c|}
\hline Cadena & Mujeres & Hombres & Nh & Wh & nh & Wh & $\begin{array}{l}\text { Media } \\
\text { estrato }\end{array}$ & $\begin{array}{l}\text { Wh media } \\
\text { estrato }\end{array}$ & $\begin{array}{c}\text { Varianza } \\
\text { estrato }\end{array}$ & $\begin{array}{c}\text { Varianza } \\
\text { estratificada }\end{array}$ \\
\hline Amaranto & 7.00 & 53.00 & 60.00 & 0.07 & 3.20 & 0.07 & 18.76 & 1.33 & 1058.00 & 313.09 \\
\hline Ornamentales & 14.00 & 50.00 & 64.00 & 0.08 & 3.41 & 0.08 & 18.76 & 1.42 & 648.00 & 179.78 \\
\hline Maíz & 12.00 & 38.00 & 50.00 & 0.06 & 2.67 & 0.06 & 18.76 & 1.11 & 338.00 & 120.03 \\
\hline Hortalizas & 20.00 & 114.00 & 134.00 & 0.16 & 7.14 & 0.16 & 18.76 & 2.98 & 4418.00 & 585.40 \\
\hline Nopal & 89.00 & 64.00 & 153.00 & 0.18 & 8.16 & 0.18 & 18.76 & 3.40 & 312.50 & 36.27 \\
\hline Bovinos & 5.00 & 25.00 & 30.00 & 0.04 & 1.60 & 0.04 & 18.76 & 0.67 & 200.00 & 118.37 \\
\hline Apícola & 26.00 & 37.00 & 63.00 & 0.07 & 3.36 & 0.07 & 18.76 & 1.40 & 60.50 & 17.05 \\
\hline Cunícola & 38.00 & 54.00 & 92.00 & 0.11 & 4.91 & 0.11 & 18.76 & 2.04 & 128.00 & 24.70 \\
\hline Ovinos & 21.00 & 69.00 & 90.00 & 0.11 & 4.80 & 0.11 & 18.76 & 2.00 & 1152.00 & 277.27 \\
\hline Porcinos & 15.00 & 45.00 & 60.00 & 0.07 & 3.20 & 0.07 & 18.76 & 1.33 & 450.00 & 133.17 \\
\hline Acuícola & 12.00 & 36.00 & 48.00 & 0.06 & 2.56 & 0.06 & 18.76 & 1.07 & 288.00 & 106.53 \\
\hline
\end{tabular}

La asignación proporcional por cadena de valor agrícola quedó de la siguiente manera: amaranto $=3.84$; ornamentales $=4.09 ;$ maíz $=$ 3.20 , hortalizas $=8.57$, nopal $=9.79$, bovinos $=$ 1.92 , apícola $=4.03$, cunícola $=5.89$, ovinos $=$ 5.76 , porcinos $=3.84$ y acuícola $=3.07$. La media fue de 46.74 , la Beta 9.61, el error permisible 0.20 y el error permisible en porcentaje 1.09

\section{DesarRollo y RESULTADOS}

Selección de extensionistas agrícolas. Con el objetivo de mejorar la eficiencia en la atención a las demandas de los productores agrícolas de la CDMX, y con base al presupuesto federal y estatal (SAGARPA 2015) se contrataron a los técnicos por un periodo de 11 meses que comprendió de abril de 
2016 a febrero de 2017, de acuerdo al Cuadro No. 3.

Cuadro 3. Extensionistas contratados por cadena de valor en la CDMX.

\begin{tabular}{|c|c|c|c|}
\hline Cadena Productiva & No. de extensionistas & Cadena Productiva & No. de extensionistas \\
\hline Amaranto & 2 & Bovinos & 2 \\
\hline Hortalizas & 5 & Apícola & 3 \\
\hline Maíz & 1 & Cunícola & 3 \\
\hline Nopal & 5 & Ovinos & 2 \\
\hline Ornamentales & 2 & Porcinos & 2 \\
\hline $\begin{array}{c}\text { Desarrollo Rural } \\
\text { Coordinadora de } \\
\text { extensionistas }\end{array}$ & 1 & Total de extensionistas & 30 \\
\hline
\end{tabular}

Fuente: Elaboración propia y archivos de SAGARPA 2014, Portal SER MEXICANO

Por razones de estrategia, conviene señalar que la cadena acuícola en la actualidad no se atiende, debido principalmente a que los productores se dedican en su mayoría a la producción de peces de ornato, mismos que no están considerados dentro de las prioridades nacionales; en su lugar, se sustituyeron por la cadena avícola, para impulsar la producción de huevo a nivel de traspatio en las zonas rurales de la CDMX, la tiene una mejor respuesta sobre todo por parte de las madres de familia.
El mayor número de extensionistas asignados a las cadenas agrícolas en la CDMX, fueron los técnicos de nopal y hortalizas, los cuáles representaron las dos terceras partes del personal que se destinó a la atención del programa de extensionismo de la SAGARPA, los cuales de manera preponderante atendieron a las Alcaldías de Milpa Alta, Tláhuac y Xochimilco y la cadena con el menor número de personal técnico fue la de maíz, ya que sólo la atendió una extensionista. La distribución porcentual se observa en la Gráfica No. 1.

\section{Extensionistas en cadenas agrícolas en la CDMX}

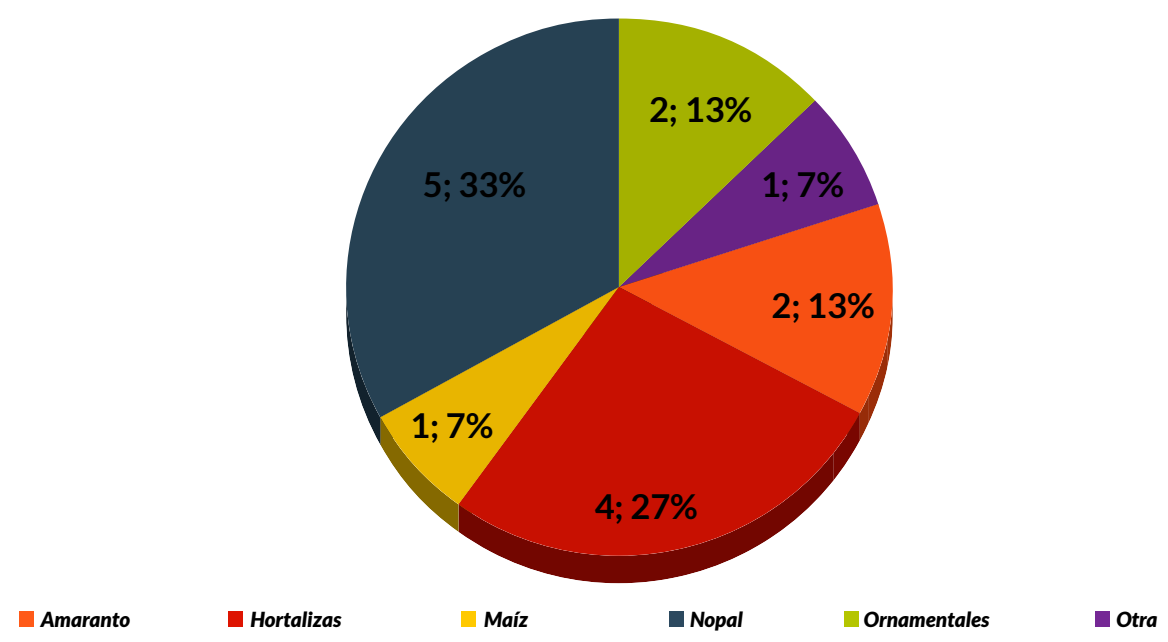

Gráfica 1. Número de extensionistas y su participación porcentual en la atención a cadenas de valor agrícolas en la CDMX. 
La participación de género (FAO 2016) dentro del componente de extensionismo agrícola de ma- nera bipartita entre la SAGARPA y la SEDEREC del Gobierno de la CDMX, se muestra en la Gráfica 2.

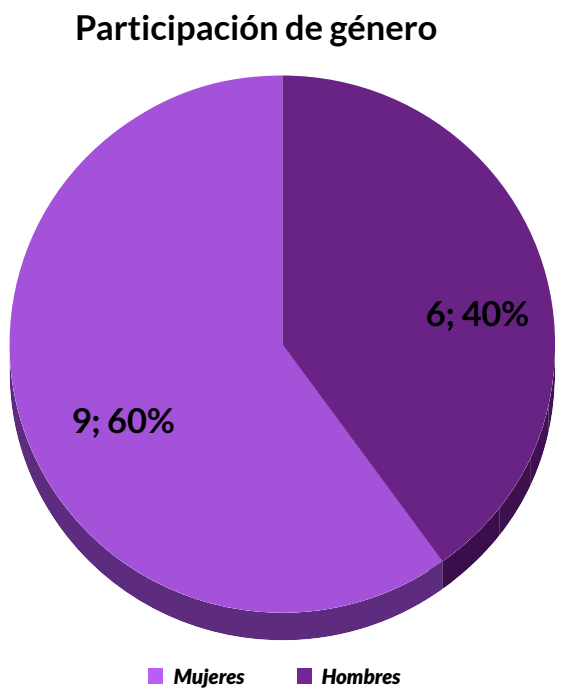

Gráfica 2. Participación de género en las cadenas agrícolas de la CDMX.

La participación de género (FAO 2016) dentro es notable dentro del extensionismo agrícola en la CDMX ya que representó el $40 \%$ de los técnicos, dentro de lo cual destacan por sus aportaciones y opiniones en el mejoramiento de las cadenas de valor agrícolas.

Con relación a los productores participantes, se solicitó por parte de SAGARPA y SEDERC a los extensionistas la conformación de un padrón de por lo menos 30 agricultores, esta cantidad se aumentó recientemente para que sean atendidos 50 productores y que estuvieran clasificados dentro de los niveles I y II de las reglas de operación del componente extensionismo para que se considerara su atención (SAGARPA 2016); con base a lo anterior el número de productores que se atendieron se muestra en el Cuadro 4.

Cuadro 4. Padrón de productores agrícolas clasificados por género y cadena de valor que se atendieron en el componente extensionismo de la CDMX.

\begin{tabular}{|c|c|c|c|}
\hline Cadena & Mujeres & Hombres & Total \\
\hline Amaranto & 7 & 53 & 134 \\
\hline Hortalizas & 20 & 114 & 50 \\
\hline Maíz & 12 & 38 & 153 \\
\hline Nopal & 89 & 64 & 64 \\
\hline Ornamentales & 14 & 50 & 30 \\
\hline Bovinos & 5 & 25 & 63 \\
\hline Apícola & 26 & 37 & 92 \\
\hline Cunícola & 38 & 54 & \\
\hline
\end{tabular}




\begin{tabular}{|c|c|c|c|}
\hline Cadena & Mujeres & Hombres & Total \\
\hline Ovinos & 21 & 69 & 60 \\
\hline Porcinos & 15 & 45 & 48 \\
\hline Acuícola & 12 & 36 & 30 \\
\hline Desarrollo Rural & 7 & 23 & $\mathbf{8 7 4}$ \\
\hline Total & $\mathbf{2 6 6}$ & $\mathbf{6 0 8}$ & \\
\hline
\end{tabular}

Fuente: Elaboración propia y archivos de SAGARPA 2014, Portal SER MEXICANO y SAGARPA 2018

El mayor número de productores que se atendieron fueron los de la cadena agroproductiva nopal, lo cual resulta lógico, en razón de que fue la cadena que tuvo un mayor de extensionistas y en consecuencia en donde se asignó el menor número de técnicos, en nuestro caso, la cadena maíz, fue la que contó con menos productores que se atendieron por cadena.

Con relación a la participación por género en el componente extensionismo agrícola de la CDMX, empieza a tener relevancia como se puede observar en la Gráfica 3.

\section{Distribución de productores por cadena y género}

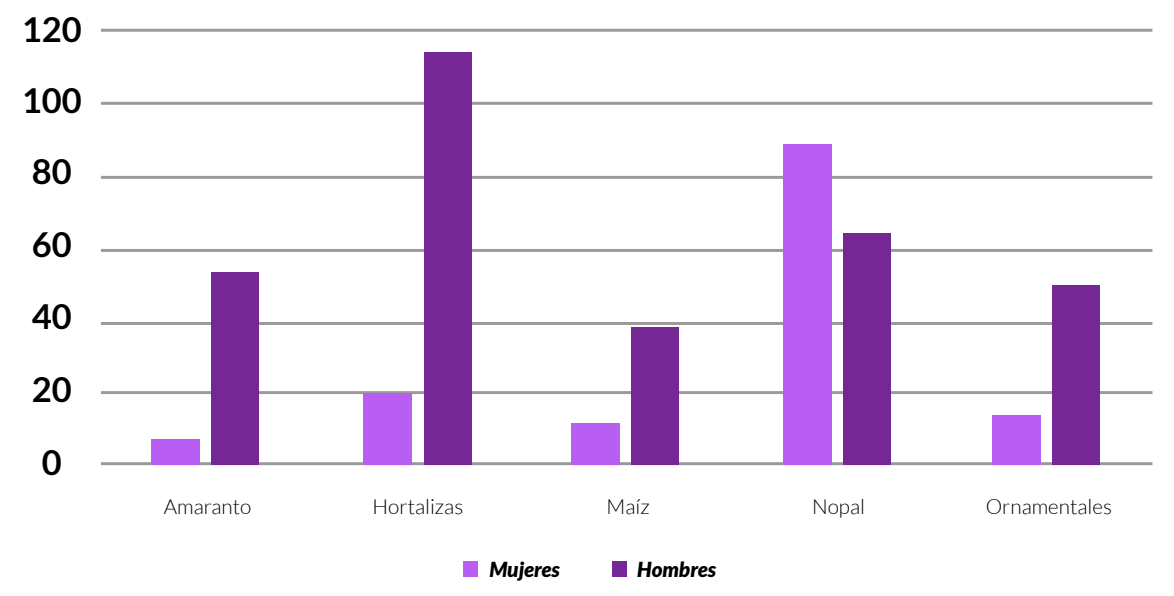

Gráfica 3. Composición de los productores por cadena agrícola y género en el programa de extensionismo de la CDMX.

La participación de la mujer en la cadena de valor nopal es de llamar la atención, ya que representan el $58.2 \%$ del total de los productores nopaleros y a nivel global en el componente extensionismo, la participación de género es de $30.8 \%$, lo cual es un reflejo de la feminización del campo a nivel de la CDMX.

Evaluación de componente extensionismo, con base a las encuestas que respondieron los productores, se obtuvieron 21, de las cuáles correspon- dieron 17 a productores líderes y 4 de productores cooperantes. El 95.24\% de los que se encuestaron tuvieron conocimiento del modelo de Red de Extensionismo e Innovación (REI), mismo que le informó el extensionista. Por otro lado, el 85.76\% de los productores participaron en algún evento de difusión, transferencia de tecnología o de capacitación y un 90.48\% participó en la implementación de alguna de las innovaciones agrícolas que fueron propuestas por los extensionistas del programa. En cuanto a la experiencia de haber compartido 
experiencias con otros productores de la localidad, el 95.24\% respondió de manera favorable y el número de productores agrícolas a los cuales se les pudo irradiar el programa de extensionismo en la CDMX fue de 427 agricultores de las cadenas que se mencionaron renglones arriba y en cuanto al dese de continuar participando en el programa de extensionismo de la SAGARPA y SEDEREC, la totalidad de productores (100\%) su interés de que se dé continuidad al programa y sigan obteniendo el apoyo de la asesoría técnica agrícola. (Santos 2018, IICA 2018).

En las preguntas de tipo cuantitativo, se utilizó una escala del $1 \mathrm{al} 10$, en la que 10 significó muy buena; 9 y 8 significó buena; 7 y 6 regular y 5 o menos se catalogó como mala. Las calificaciones se muestran en el Cuadro No. 5 .

\begin{tabular}{|c|c|c|c|c|}
\hline Pregunta & Valor max & Valor min & Promedio & Moda \\
\hline Cómo califica el modelo REI & 10 & 7 & 8.90 & 8 \\
\hline $\begin{array}{c}\text { Cómo califica las innovaciones } \\
\text { implementadas }\end{array}$ & 10 & 7 & 8.95 & 10 \\
\hline $\begin{array}{l}\text { Cómo califica los beneficios que obtuvo } \\
\text { de las innovaciones implementadas }\end{array}$ & 10 & 8 & 9.10 & 9 \\
\hline $\begin{array}{l}\text { Cómo califica la participación del } \\
\text { extensionista en el modelo REI }\end{array}$ & 10 & 7 & 9.10 & 10 \\
\hline $\begin{array}{l}\text { Cómo califica la información } \\
\text { que recibió con relación al } \\
\text { extensionismo(socialización de } \\
\text { agendas, giras tecnológicas, recorridos } \\
\text { de campo, capacitaciones, charlas }\end{array}$ & 10 & 8 & 9.29 & 9 \\
\hline
\end{tabular}

Fuente: Elaboración propia con datos de las encuestas.

Del anterior cuadro se destaque el que, las calificaciones más frecuentes (las modas) fueron para las variables de las innovaciones implementadas y la de la participación del extensionista, sin embargo, las valoraciones más bajas (mínimos) se dieron también en las innovaciones y en la participación del extensionista y también se le suma la del modelo REI.

Las principales innovaciones agrícolas que se implementaron con el programa de extensionismo de la SAGARPA y la SEDEREC en la CDMX, bajo la supervisión de los extensionistas fueron las siguientes: manejo de abonos orgánicos, caldos minerales, lombricomposta, semillas mejoradas, inoculación, siembra de conservación, micorrizas, filtros para limpiar agua, repelentes para plagas, composteo, biofertilización, desarrollo de nuevos productos, plan de negocios, diseño de imagen corporativa, bitácoras técnicas y económicas, fertilización foliar, certificación y acolchado.
Con relación a los beneficios que se detectaron por los productores agrícolas se mencionan los siguientes: reducción de costos y mayores rendimientos, mayor ingreso, obtención de conocimiento, menor consumo de agroquímicos y fertilizantes químicos, mayor producción en temporadas críticas, transformación de productos, mayores mercados, mejores ventas, menor contaminación del medio ambiente, mejor administración, vinculación con otras empresas, mayor capacidad de gestión y cooperativismo, mejor calidad de los productos y mayor competitividad. La encuesta de tipo estructurada con preguntas abiertas (cualitativas) y mediante valoraciones numéricas (cuantitativas) que se implementó, tuvo un error permisible medido en porcentaje del $9 \%$, con lo cual se considera una encuesta aceptable.

\section{Conclusión}

El programa de extensionismo en la CDMX fue la cuarta vez que operó y el primer año (2016) en 
el que se suma el INIFAP y ya son tres años de manera consecutiva en los que participa y el enfoque principal de la investigación en el sentido de sí se continúa o no, con el componente extensionismo en la CDMX, la evaluación que realizaron los productores primarios fue que el programa continúe en razón de los beneficios que obtienen y es así que el 90.5\% de los campesinos participantes implementaron por lo menos una innovación bajo la conducción y supervisión de los extensionistas agrícolas y el compartir las experiencias con otros productores de sus localidades, expandió el conocimiento. Finalmente, los principales beneficios que señalaron los productores que implementaron innovaciones fueron los de mayores rendimientos, menores costos, mejor calidad, transformación de sus materias primas para dar un valor agregado, mayor capacidad de gestión y concientización para asociarse (cooperativismo) 


\section{Referencias bibliográficas}

Aguilar A.J., Altamirano C. J. R. y Rendón M. R. 2010. Del Extensionismo Agrícola a las Redes de Innovación Rural. Universidad Autónoma Chapingo. Estado de México, México. 281 pp. http://www.redinnovagro.in/documentosinnov/ extensionismo_30_sept.pdf consulta abril 2018.

Cadena I. P. Guerra M. M., González C. M., Berdugo R. J. G. y Ayala S. A. 2009. Estrategias de Transferencia de Tecnología, como Herramientas del Desarrollo Rural. INIFAP. Centro de Investigación Pacífico Sur. Campo Experimental Centro de Chiapas. Libro Técnico No. 2. Ocozocoautla de Espinosa, Chiapas, México. 112 pp.

Cadena I.P., Camas G. R., Rodríguez H. R. F., Berdugo R. J. G., Ayala S. A., Zambada M. A., Morales, G. M., Espinoza P. N. y López B. W. 2015. Contribuciones del INIFAP al Extensionismo en México y la Gestión de la Innovación. Revista Mexicana de Ciencias Agrícolas. Vol. 6. No. 4. Chapingo, Estado de México. México. 883 - 895 pp

Comisión Nacional de Población. 2015. Anuarios Estadísticos. CDMX. México.

Cuevas R. V., Becerra L. F., Vázquez G. R. y Tapia N. C. A. 2013. La Investigación Agropecuaria, Forestal y sus Esfuerzos de Transferencia y Divulgación Mediante Esquemas de Extensión. El Servicio de Extensión Rural en México. Propuestas de Política Pública. Editorial BBA. 109 - 129 pp

Cuevas R. V., J. Baca del M. y J. Sánchez G. 2012. Actores en el desarrollo territorial rural: elementos relevantes y redes de conocimiento de los extensionistas pecuarios en Sinaloa, México. Spanish Journal of Rural Development. Vol. III (4) 63 $-78$

Cuevas R. V., Vázquez G. R. y Landa F. E. 2017. Demandas de capacitación tecnológica de extensionistas y productores pecuarios participantes en el componente extensionismo. Memoria. Reunión Nacional de Investigación Pecuaria. Ciencia y Tecnología para la Ganadería Tropical Mexicana, Acapulco, Gro. México. 832 pp

Diario Oficial de la Federación. 13 diciembre 2013. Decreto por el que aprueba el Programa Sectorial de Desarrollo Agropecuario, Pesquero y Alimentario 2013 - 2018.

Diario Oficial de la Federación. 30 diciembre 2015. Acuerdo por el que se dan a conocer las Reglas de Operación de los Programas de la Secretaría de Agricultura, Ganadería, Desarrollo Rural, Pesca y Alimentación para el ejercicio fiscal 2016.

Food Agricultural Organization. 2016. Buscan reducir la brecha de género en los programas de SAGARPA. FAO en México. www.fao.org/mexico/noticias/detail-events/es/c/420797/

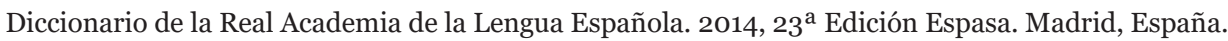

Espinosa G. J. A., A. Vélez I., M. Borja B., R. Vázquez G. y G. Moctezuma L. 2017. La Participación de las Instituciones de Investigación y Enseñanza Superior en el Componente de Extensionismo en 12 Estados del Centro de México. XVII. Encuentro Nacional Académico en Administración de Agronegocios. Memoria. Ciudad de México, México. 17 - 31 pp

Gregoire, Timothy G., and HarryT. Valentine. 2008. Sampling Strategies for Natural Resources and Environment. Boca Raton, Fl.: Chapman and Hall / CRC. https://www.crcpress.com/Sampling-Startegies-for-Natural-Resources-and-theEnvironment/Gregoire-Valentine/p/book/9781584883708.

Herrera Tapia Francisco. 2013. Enfoques y políticas del desarrollo rural en México. Una Revisión de su Construcción Institucional. Gestión y Política Pública. Vol. XXII. Num. 1. 131 - 159 pp

Instituto Interamericano de Cooperación para la Agricultura. Instituto Nacional de Capacitación Rural. 2012. Extensionismo y gestión territorial para el desarrollo rural. Distrito Federal, México. 192 pp

Instituto Interamericano de Cooperación para la Agricultura. 2018. Extensionismo con Resultado. Estrategia de Política Pública y Sistematización de Experiencias de Extensionismo Rural con Enfoque Territorial. Ciudad de México. México. $118-122 \mathrm{pp}$

Instituto Nacional de Geografía y Estadística. Estados Unidos Mexicanos. (2014) Distrito Federal. Aguascalientes, México.

Instituto Nacional de Geografía y Estadística. Estados Unidos Mexicanos. (2015) Censo Agrícola, Ganadero y Forestal. Aguascalientes México.

Manrubio M. R., Altamirano C. J. Reyes., Aguilar A. J., Rendón M. R., García M. J. G. y Espejel G. A. 2007. Innovación: Motor de la Competitividad Agroalimentaria - Políticas y Estrategias para que en México Ocurra - CIESTAAM. Universidad Autónoma Chapingo. Texcoco, Estado de México. 310 pp. 
McMahon M.A., Valdés A., Cahill C. y Jankowska C. 2011. Análisis del Extensionismo Agrícola en México. OCDE. 50 Mejores Políticas para una Vida Mejor. Paris, Francia. 73 pp.

Méndez Sastoque Marlon Javier. 2006. Los Retos de la Extensión ante una Nueva y cambiante Noción de lo Rural. Rev. Fac. Nac. Agron. Medellín. Colombia. Vol. 59. No. 2. 3407 - 3423.

Moctezuma, L. G, J. A. García E., V. Cuevas R., J. L. Jolalpa B y F. Romero S. 2008. Detección de Demandas de Investigación Tecnológicas, de Conocimiento y de Política en la Cadena Agroalimentaria Cebada en el Estado de Hidalgo, México. XLIV Reunión Nacional de Investigación Pecuaria y III Reunión Nacional de Innovación Agrícola y Forestal Yucatán 2008. Memoria. Guadalajara, Jal. México. 247 p

Moctezuma L. G., Velázquez F. L., González C. G. y Castillo C. A. L. 2016. Avances del Extensionismo Pecuario en la CDMX. Memoria de la Reunión Nacional de Investigación Pecuaria. INIFAP. Querétaro. Qro. 516 - 518 pp

Moctezuma L. G., Velázquez F. L. y Ramírez S. E. U. 2018. Importancia del Extensionismo Agropecuario en la Ciudad de México (CDMX). Memoria. Seminario Internacional de Investigación "Experiencias y Perspectivas de la Investigación en América Latina”. Universidad Autónoma Chapingo, Chapingo, Estado de México. México 20 p

Rendón M. R., E. Roldán S., B. Hernández H. y P. Cadena I. 2015. Los procesos de Extensión rural en México. Revista Mexicana de Ciencias Agrícolas. Vol. 6. Num. 1. $151-161$ pp

Royo. M. M. H., J. S. Sierra T., J. O. Ronquillo O., J. M. Ochoa. R. y R. Vázquez G. 2018. Análisis del componente extensionismo SAGARPA - INIFAP (2016) en el norte de México. Revista Mexicana de Ciencias Agrícolas. Vol. 8. Núm. 4 $813-826 \mathrm{pp}$

Saldaña, A. R., J. A. Espinosa G., G. Moctezuma L., A. Ayala S., C. A. Tapia N., R.M. Ríos I., S. M. Valle L. y A. M. Gomes de C. 2006. Proyecto Quo Vadis: El Futuro de la Investigación Agropecuaria y Forestal y la Innovación Institucional de México. INIFAP. México, D. F. 276 pp

Sanderson S.E. La Transformación de la Agricultura Mexicana. Estructura Internacional y Política del Cambio Rural. 1990. Consejo Nacional para la Cultura y el Arte. Alianza Editorial Mexicana. México, D. F. 290 pp

Santos C. V. M. y A. Álvarez M 2018. Análisis de Políticas de Extensionismo en México: hacia un Nuevo Extensionismo Basado en Evidencia Empírica. ${ }^{\text {a }}$ Jornada de Trabajo e Investigación Científica. Universidad Autónoma Metropolitana. Ciudad de México, México. 105 pp

Santoyo C. V. H. y E. G. Martínez G. 2017. La Investigación y la Educación Superior en el Nuevo Extensionismo. 2017. XVII. Encuentro Nacional Académico en Administración de Agronegocios. Memoria. Ciudad de México, México. 6 - 16 pp

Secretaría de Agricultura, Ganadería Desarrollo Rural, Pesca y Alimentación y Organización de las Naciones Unidas para la Alimentación y la Agricultura. 2014. Compendio de Indicadores de Gestión y Resultados 2014. Componente de Extensión e Innovación Productiva del Programa Integral de Desarrollo Rural. Ciudad de México, México. 99 pp

Secretaría de Agricultura, Ganadería Desarrollo Rural, Pesca y Alimentación y Organización de las Naciones Unidas para la Alimentación y la Agricultura. 2015. Compendio de Indicadores de Gestión y Resultados 2015. Componente de Extensión e Innovación Productiva del Programa Integral de Desarrollo Rural. Ciudad de México, México. 85 pp

Secretaría de Agricultura, Ganadería Desarrollo Rural, Pesca y Alimentación. 2015. Convenio de Coordinación para el Desarrollo Rural Sustentable 2015 - 2018. Durango, México. 22 pp

Secretaría de Agricultura, Ganadería Desarrollo Rural, Pesca y Alimentación y Organización de las Naciones Unidas para la Alimentación y la Agricultura. 2016. Compendio de Indicadores de Gestión y Resultados 2016. Componente de Extensión e Innovación Productiva del Programa Integral de Desarrollo Rural. Ciudad de México, México. 100 pp

Secretaría de Agricultura, Ganadería Desarrollo Rural, Pesca y Alimentación. 2007. Programa Nacional Pecuario. México, D. F. $42 \mathrm{pp}$

Secretaría de Agricultura, Ganadería Desarrollo Rural, Pesca y Alimentación. SER MEXICANO (2014). www.extensionismo. $\mathrm{mx} /$ web1/index.php/contenido/119-extensionismo-rural

Secretaría de Agricultura, Ganadería Desarrollo Rural, Pesca y Alimentación.2016. Reglas de Operación. URL disponible en http://www.sagarpa.gob.mx/agricultura/Programas/proagro/Normatividad/Documentos/2016/Reglas_ Operación_2016_sagarpa_DOF_Oficial.pdf

Secretaría de Agricultura, Ganadería Desarrollo Rural, Pesca y Alimentación. 2018. Anexo Técnico de Ejecución Específico para el Desarrollo Presupuestal 2018 de la Ciudad de México. México. 16 pp 
Secretaría de Agricultura, Ganadería Desarrollo Rural, Pesca y Alimentación y Secretaría de Desarrollo Rural y Equidad de las Comunidades. 2018.Publicación de Resultados de la Dictaminación a las Solicitudes del Programa de Concurrencia con las Entidades Federativas. Ejercicio 2018. Componente Infraestructura, Equipamiento, Maquinaria y Material Biológico. Ciudad de México, México. 1 p

Secretaría de Agricultura, Ganadería Desarrollo Rural, Pesca y Alimentación. 2018. Programa de Apoyo a Pequeños Productores, en su Componente de Extensionismo, Desarrollo de Capacidades y Asociatividad Productiva. Ejercicio 2018. Ciudad de México. México. 2pp

Sistema de Información Agrícola y Pecuaria. SAGARPA Anuario Estadístico de la Producción Pecuaria. 2013.

Sistema de Información Agrícola y Pecuaria. SAGARPA Anuario Estadístico de la Producción Pecuaria. 2014.

Sistema de Información Agrícola y Pecuaria. SAGARPA Anuario Estadístico de la Producción Pecuaria. 2015.

Solleiro R. J. L., R. Castañón I., J. D. González C., J. Aguilar A. y N. Aguilar G. 2017. Identificación de Buenas Prácticas de Extensionismo, Transferencia de Tecnología e Innovación para el Sector Agroalimentario de México. XVII Congreso Latino Iberoamericano de Gestión Tecnológica. Gestión de la Innovación para la Competitividad: Sectores Estratégicos, Tecnologías Emergentes, y Emprendimientos. Ciudad de México, México. 1 - 15 pp

Solleiro R. J. L., R. Castañón I. y J. D. González C. 2017.Análisis de políticas públicas en materia de extensionismo y transferencia de tecnología del sector agroalimentario en México y recomendaciones para su fortalecimiento. Economía y Desarrollo. Vol. 158. Ciudad de México, México. 50 - 65 pp

Reyes Osorio Sergio. 2013. El Servicio de Extensión Rural en México. Propuesta de Política Pública. Biblioteca Básica de Agricultura, México. 156 pp

Turrent F. A. Políticas de Investigación y Transferencia Agrícola, Pecuaria y Forestal para el Campo Mexicano. 2007. En Desarrollo Agropecuario y Forestal. Agenda para el Desarrollo. Volumen 9. Editorial Miguel Ángel Porrúa. México, D. F. $135-146$ PP.

Vázquez G. R. y Landa F. E. 2017. Relación entre el perfil técnico de los extensionistas pecuarios y su desempeño. Memoria. Reunión Nacional de Investigación Pecuaria. Ciencia y Tecnología para la Ganadería Tropical Mexicana, Acapulco, Gro. México. $832 \mathrm{pp}$

Villareal René. 2015. Extensionismo Holístico y el Agrocluster. Modelo de Asociatividad e Integración de la Cadena Productiva. SAGARPA. México. 38 pp.

Wikipedia. Enciclopedia libre. 2016. es.wikipedia.org/wiki/México-Tenochtitlan.

Zamora M. M. C. 2016. Extensionismo Forestal. Revista Mexicana de Ciencias Forestales. Vol.7. No. 36. Julio-agosto. México. 4- 5 pp. 
Revista incluida en los siguientes agregadores de contenidos
Revista incluida en los siguientes directorios

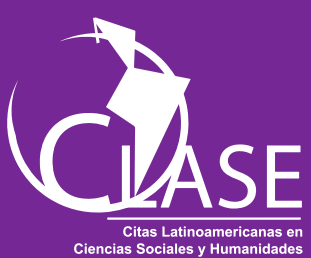

llboblat

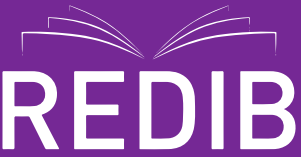

Red Iberoamericana

de Innovación y Conocimiento Cientifico

\section{Google Académico}
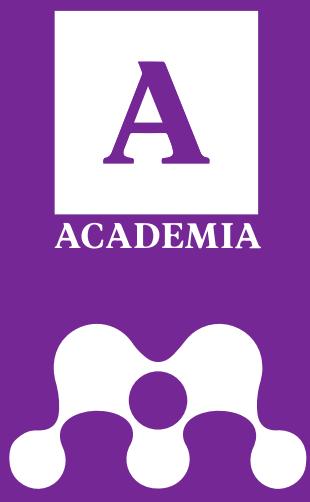

MENDELEY

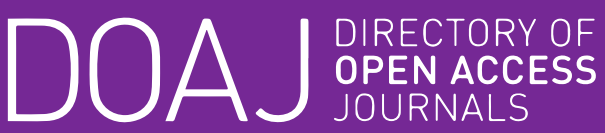

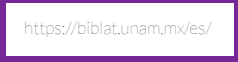
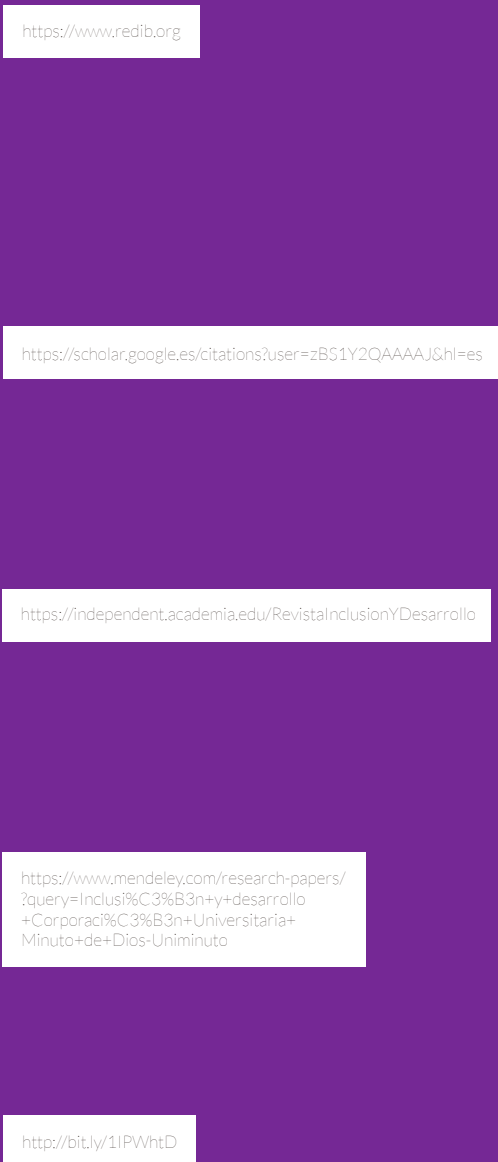

Revista incluida en la siguiente red social

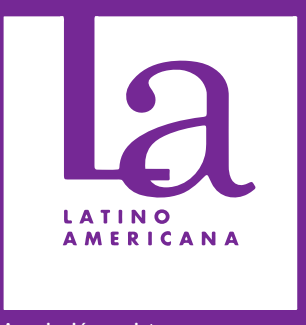



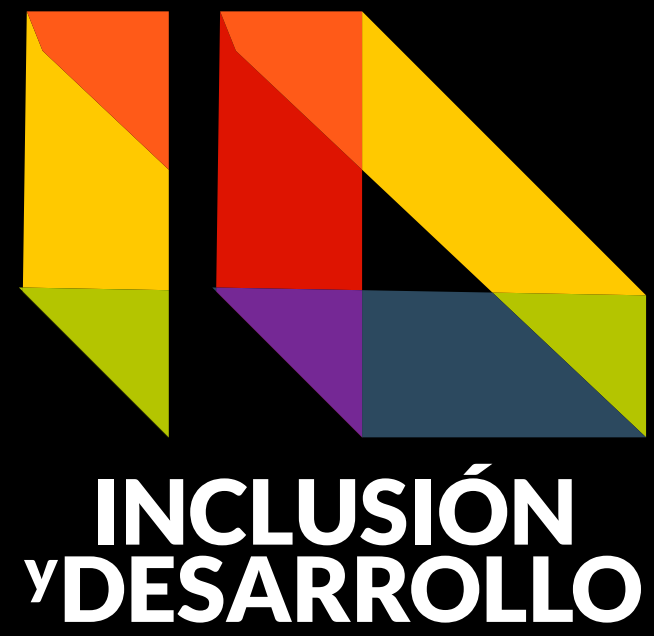

No. 1 Vol. 6 Año 2019 ISSN En línea: 2590-7700

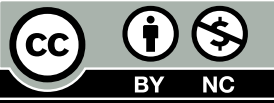

